\title{
Early Post-Operative Complications in Surgeries Pertaining Oral and Maxillofacial Region in MNH, Tanzania
}

\author{
Karpal Singh Sohal ${ }^{*}$, Farid Shubi ${ }^{2}$ \\ ${ }^{1}$ Department of Oral and Maxillofacial Surgery, MNH, Dar es Salaam, Tanzania \\ ${ }^{2}$ Department of Oral and Maxillofacial Surgery, MUHAS, Dar es Salaam, Tanzania \\ Email: ${ }^{*}$ karpal@live.com
}

Received 4 September 2015; accepted 25 October 2015; published 28 October 2015

Copyright (C) 2015 by authors and Scientific Research Publishing Inc.

This work is licensed under the Creative Commons Attribution International License (CC BY). http://creativecommons.org/licenses/by/4.0/

(c) (i) Open Access

\section{Abstract}

The Oral and maxillofacial region is one of the complex regions of the body considering the vast anatomical structures that are found in this region; head and neck surgery has potential for many complications, yet inadequate information on occurrence of post-operative complications in oral and maxillofacial surgeries in our setting has been documented. The aim of this study was to determine the occurrence of common early post-operative complications after oral and maxillofacial surgery in relationship to the underlying systemic condition. A descriptive postoperative study was done whereby patients who underwent oral and maxillofacial surgery were included. The included patients were those who underwent surgery for different pathological conditions, trauma, developmental/congenital conditions and inflammatory conditions to mention few. Demographic data, complications developed within one week post operative, and underlying systemic comorbidities before and after surgery were documented and analysed. A total of 102 patients were included in the study. The mean age of participants was $30.00 \pm 17.01$ years with a range of 2 to 81 years. Majority $43.1 \%(n=44)$ had benign tumors with a leading diagnosis of ameloblastoma. In general, the most common complications which were noted in the cases included Pain $98 \%$ ( $n=$ 100) and Post-operative Swelling $97.1 \%(n=99)$. The presence of underlying systemic comorbid conditions has a significant role in occurrence of some severe complications. The occurrence of complications does increase the duration of stay in the hospital hence increasing cost of treatment for which bearers are both patients and the hospital.

\section{Keywords}

Early Post-Operative Complication, Oral and Maxillofacial Surgery, Systemic Conditions

\footnotetext{
${ }^{*}$ Corresponding author.
} 


\section{Introduction}

Surgery is an ancient medical specialty that uses operative manual and instrumental techniques on a patient to investigate and/or treat a pathological condition such as disease or injury, or to help improve bodily function or appearance [1].

Oral and maxillofacial region is one of the complex regions of the body, considering the vast anatomical structures that are found in this region. The head and neck region of the body contains many important structures compressed into a relatively small area [2]. These structures comprise of bones, neurovascular structures, different glands (like salivary glands, lacrimal glands etc.), different muscles, eyes, nose and many more not to forget the skin.

There are numerous conditions that may occur in the oral and maxillofacial region, ranging from developmental defects, injuries, and different pathologies including both benign and malignant diseases to the functional and esthetic aspects of the hard and soft tissues.

Despite several treatment modalities for treating the conditions that arise in the oral and maxillofacial region, surgical approach remains one of the essential methods for managing these conditions [3]. Some of the surgical procedures that are carried out in this region include tumor excision, mandibulectomy and maxillectomy (partial, hemi- or total), open reduction and internal fixation (ORIF) of bone fractures, and reconstruction surgeries to name the few.

Surgical outcome are more or less predictable with a reasonable degree of accuracy. None of the procedures being performed in routine head and neck surgery are novel. Head and neck surgery has potential for many complications due to presence of large number of vessels and nerves [4].

A surgical complication has been defined as a development which is generally to the patient's detriment arising at the time of operation or during the post-operative period [5]. The surgical complication may occur, either at the time of surgery or in the post-operative period, they might be progressive, or may occur later, months or even years after the operation [6].

The objective of this study was to determine the common early post-operative complications after oral and maxillofacial surgery in relation to the systemic condition, disease condition and demographic characteristic of the patient.

\section{Materials and Methods}

The medical records of patients who underwent maxillofacial surgery between April 2014 and November 2014 were reviewed for the occurrence of complications. Informed consent for surgery was obtained from the patients or their parents/caretakers.

All the patients who consented and underwent surgery in maxillofacial region for different pathological conditions, trauma, developmental/congenital conditions and inflammatory conditions were included in the study. Any complications occurring to the patient within 7 days post-surgery, such as wound dehiscence, conjunctivitis, pain etc. were recorded in the dataset designed for the study. Complications within one week (7 days) of surgery were regarded as early complications.

Surgical procedures were performed under general anesthesia. The patients were operated using extra oral, intra oral or both extra and intra oral routes. Surgical procedures employed included recessions, resections, open reduction and internal fixation of fractures (ORIF), enucleation, and bone remodeling. Absorbable sutures were used for deeper and intra-oral tissue repair, while non-absorbable ones were used for skin. Parental antibiotics and analgesics were given in first 5 days followed by oral ones for 5 days; steroids were given for the first 48 72 hours post op. The patients were evaluated on postoperative days 1,3 , and 7 for any complications. Data collected was coded, transferred to the computer and were analyzed by chi-square test and values were considered to be significant if $\mathrm{P}<0.05$, by using the SPSS computer program version 19 . Permission to undertake the study was granted by the Department of Oral and Maxillofacial Surgery of MNH.

\section{Results}

A total of 102 patients, who underwent maxillofacial surgical procedures were included in this study. The age range of the participants ranged from 2 years to 81 years, the mean age being $30.00 \pm 17.01$, with $26.5 \%(n=27)$ of the patient falling in the age group of 20 - 29. Of the 102 patients who were included in this study, $57.8 \%(\mathrm{n}=$ 
59) were male, with the male to female ratio being 1.4:1 (Table 1).

The duration of stay in the hospital post-operative, ranged from 3 days to 41 days, with mean duration of stay being $8.58 \pm 6.75$.

Of the all cases that were surgically treated, $43.1 \%(n=44)$ accounted for benign lesions (Table 2). The common benign lesions to be operated were ameloblastoma $(n=11)$ and ossifying fibroma $(n=10)$. Sarcomas $(n=6)$ were most surgically treated malignant lesion followed by squamous cell carcinoma $(n=3)$. In the category of traumatic conditions, most cases had mandibular fractures $(n=9)$ and both midface and mandibular fractures $(n=7)$.

With the exception of Traumatic cases, in all other cases there was more predominance of female patients than male counter parts.

Most of the incisions that were made were extra oral $(54.9 \%, \mathrm{n}=56)$ followed by intra-oral approach $(36.3 \%$, $\mathrm{n}=37)$, while 8 cases $(8.8 \%, \mathrm{n}=9)$ had both intra and extra oral approach. There was no significant difference observed in occurrence of different complications in relation to site of incision in general, except for esthetic impairment and functional impairment ( $\mathrm{p}=0.001$ and 0.012 respectively).

The most common complications which were noted in the cases included Pain $(98 \%, n=100)$ and Post-operative Swelling $(97.1 \%, n=99)$, while the least common ones were hiccups $(3.9 \%, n=4)$ and convulsions $(1 \%$, $\mathrm{n}=1$ ) (Table 3).

Of the 102 patients who were included in this study, $11.8 \%(n=12)$ had some underlying systemic/medical condition that included anemia, HIV/AIDS, Hypertension, Diabetes mellitus, and/or malnutrition. The presence of underlying systemic condition had significant effect on occurrence of surgical site infection $(p=0.000)$, other complications with significant difference observed when comparing between those patients with underlying medical conditions to those without included speech impairment, wound dehiscence, functional impairment, vomiting, and convulsions were concerned (Table 4).

Table 1. Distribution of the patients by age group and gender.

\begin{tabular}{ccccc}
\hline & & Gender & & Total \\
\cline { 2 - 4 } Age group & Male & Female & 10 \\
$10-19$ & 5 & 5 & 18 \\
$20-29$ & 12 & 6 & 12 & 27 \\
$30-39$ & 15 & 7 & 18 \\
$40-49$ & 11 & 6 & 8 \\
$50-59$ & 9 & 4 & 6 \\
$60+$ & 4 & 3 & $\mathbf{1 0 2}$ \\
\hline
\end{tabular}

Table 2. Distribution of the nature of condition in relation to gender.

\begin{tabular}{|c|c|c|c|c|}
\hline \multirow{2}{*}{ Condition } & \multicolumn{2}{|c|}{ Gender } & \multirow{2}{*}{ Total } & \multirow{2}{*}{ Total Percentage (\%) } \\
\hline & Male & Female & & \\
\hline Benign lesions & 19 & 25 & 44 & 43.1 \\
\hline Malignant lesions & 7 & 11 & 18 & 17.6 \\
\hline Trauma & 27 & 0 & 27 & 26.5 \\
\hline Infection/inflammatory & 2 & 2 & 4 & 3.9 \\
\hline Others & 4 & 5 & 9 & 8.8 \\
\hline Total & 59 & 43 & 102 & 100 \\
\hline
\end{tabular}


Table 3. Distribution of the complications of the conditions that were treated surgically.

\begin{tabular}{ccc}
\hline Complication & Number of cases & Percentage (\%) \\
\hline Pain & 100 & 98 \\
Post-Operative Swelling & 99 & 97.1 \\
Functional Impairment & 24 & 23.5 \\
Aesthetic Impairment & 17 & 16.7 \\
Speech & 15 & 14.7 \\
Wound Dehiscence & 12 & 11.8 \\
Neurological Impairment & 8 & 7.8 \\
Bleeding & 8 & 7.8 \\
Surgical Site Infection & 6 & 5.9 \\
Chemical Conjunctivitis & 5 & 4.9 \\
Nausea \& Vomiting & 5 & 4.9 \\
Hiccup & 4 & 3.9 \\
Convulsion & 1 & 1 \\
\hline
\end{tabular}

Table 4. Distribution of the complication of surgery by underlying medical condition.

\begin{tabular}{cccc}
\hline \multirow{2}{*}{ Complication of surgery } & \multicolumn{2}{c}{ Underlying Medical Condition } & p-value \\
\cline { 2 - 3 } Pain & Yes & No & 0.602 \\
Post-Operative Swelling & 12 & 88 & 0.521 \\
Chemical Conjunctivitis & 12 & 87 & 0.402 \\
Bleeding & 0 & 5 & 0.946 \\
Speech impairment & 1 & 7 & 0.005 \\
Wound dehiscence & 5 & 10 & 0.014 \\
Functional impairment & 4 & 8 & 0.000 \\
Neurologic deficit & 9 & 15 & 0.282 \\
Nausea \& Vomiting & 0 & 8 & 0.044 \\
Aesthetic impairment & 2 & 3 & 0.099 \\
Surgical Site Infection & 4 & 13 & 0.000 \\
Convulsions & 4 & 2 & 0.006 \\
Hiccups & 1 & 0 & 0.402 \\
\hline
\end{tabular}

Age in general had no significant correlation to the occurrence of the early post-surgery complications; nevertheless, as far as wound dehiscence and functional impairment were of concern, increasing age had a direct effect $(p=0.000)$.

\section{Discussion}

Surgical procedures in Oral and Maxillofacial region can either be carried out under local anesthesia or general anesthesia. This study was carried out with aim of determining the early post-operative complications of surgical procedures done in Oral and Maxillofacial area under general anesthesia. The complications noted were those occurring within seven days of surgery.

There were more male patients $57.8 \%(n=59)$ when compared to the female counterparts who underwent 
surgical procedures, and this may be qualified by the fact that traumatic cases (accounting for $26.5 \%$ of all cases) which were treated during time of study were predominated by male patients entirely, and studies report trauma to have been more prevalent in males [7] [8].

The complications which were noted as an adverse effect of general anesthesia in this study were nausea \& vomiting and convulsions. Post-operative nausea and vomiting (PONV) has been associated with both intravenous and inhalation anesthetic agents. A study in rats suggested that nitrous oxide causes stimulation of the medullary periventricular dopaminergic system, which includes the CTZ, and this could be responsible for the nausea and vomiting observed after nitrous oxide anaesthesia in humans [9]. However opioids also, which are used to augment the effect of anesthesia have an effect on the chemoreceptor trigger zone in area of postema [10]. POVN was managed by use of Metoclopramide.

Anesthesia-induced convulsion/seizures are rare, and the underlying basic neurophysiological principles remain mysterious [11]. Seizures occur when cortical activity changes from its typical mode of independent and dynamically reactive activity to overwhelming synchronous oscillations in populations of neurons [11]. There are studies that document convulsions during or shortly after general anesthesia [12]-[15]. In the current study, there was a single case of post anaesthesia seizure which occurred about 4 hours post-surgery and continued for duration of 2 days. Patient was managed by intravenous injection of diazepam.

Pain was the most common post-operative complication in this study ( $98 \%$ of all patients). Pain is defined by the International Association for the Study of Pain as an unpleasant sensory and emotional experience associated with actual or potential tissue damage [16]. Almost all surgical procedures are followed by pain [17]. Tissue injury causes cellular disruption, resulting in escape of intracellular potassium ions $(\mathrm{K} p)$ and the release of bradykinin (BK), serotonin (5-HT), and prostaglandin (PG), all of which are potent activators of the peripheral endings of nociceptive neurons [18]. Optimal treatment of post-operative pain is mandatory in order to enhance recovery and reduces morbidity [17]. Gender of the patient, age and underlying medical condition had no significant difference in manifestation of postoperative pain. Pethedine, Morphine, Tramadol and Diclofenac were used to manage the pain arising post-op.

Hiccups are involuntary spasmodic contraction of diaphragm and intercostal muscles. The pathogenesis of postoperative hiccups is not known [19]. Triggering events may include distention of the stomach during bagvalve-mask ventilation. Hiccups were not very common complication in patients who underwent maxillofacial surgery in our setting, and it was managed by simple measures of vagal stimulation by drinking fluid rapidly.

Postoperative bleeding should always be considered surgically related until proven otherwise [20]. The incidence of postoperative bleeding in this study was $7.8 \%$ which is higher than in other studies which reported the incidence of less than 2\% [21] [22]. There was no significant difference in occurrence of bleeding as far as age, gender and underlying medical condition were concerned. In this study, postoperative bleeding occurred within 24 hours in almost all cases. The bleeding which occurred in the patient were almost all from surgical wound except for one case in which facial artery was the cause. The bleeding was controlled by compression alone in 5 cases, compression and administration of tranexamic acid in 2 cases and in one case the wound had to be revisited and the bleeding vessel ligated after failure to arrest bleeding by compression.

In current study, of the 102 patient recruited, $11.8 \%(n=12)$ had developed wound dehiscence, similar to some studies reporting prevalence of $0.2 \%$ - 10\% [23] [24], and low compared to a study which reported $45 \%$ [2]. Wound dehiscence/impaired healing can be contributed by multiple factors that can increase risk of wound complications, such factors include radiotherapy, chemo-radiotherapy, malnutrition, duration of surgery, anemia, tobacco use, medical comorbidity, and others [17] [25]-[27]. Age and gender had no correlation to the occurrence of wound dehiscence in this study, however under-laying medical comorbidities had significant influence on occurrence of wound dehiscence $(\mathrm{p}=0.014)$, with anemia and malnutrition playing role in most of patients. Wound dehiscence was managed by daily wound care, dressing with antiseptic medications and managing the underlying systemic condition. Wound was left to heal by secondary intention.

Surgical Site Infection (SSI) is one of the complications anticipated in surgery done on head and neck region. SSIs are defined as infections occurring within 30 days after a surgical operation (or within one year if an implant is left in place after the procedure) and affecting either the incision or deep tissue at the operation site [28]. Incidence of SSI has been reported to vary widely between procedures, hospitals, surgeons and between patients, however it occurs in about $1 \%-38 \%$ of cases in different literature [29]. In this study, incidence of SSI was $5.9 \%$, and the underlying systemic condition had significant influence in occurrence of SSI $(p=0.000)$. SSI had been noted between 4 - 7 days post-op in the cases. Management of SSI included broad spectrum antibiotic ad- 
ministration followed by specific antibiotic after culture and sensitivity of pus was done, daily irrigation and dressing of surgical site with antiseptics.

Post-Op swelling is attributed to the effect of inflammatory process. Surgery involves insult to the tissues, and thus inflammatory process occurs as a protective measure to control the cause of tissue harm to the body. The inflammation which sets-in post op is acute type, and occurs within minutes to hours post-op. After surgery, cascade of inflammation starts with micro vascular changes occurring (vascular dilation) and increased blood flow. As the microvasculature becomes more permeable, protein-rich fluid moves into the extravascular tissues, the loss of protein-rich fluid into the perivascular space reduces the intravascular osmotic pressure and increases the osmotic pressure of the interstitial fluid resulting into outflow of water and ions into the extravascular tissues, leading to a swelling. In the current study, $99.7 \%$ of the all patients who underwent surgery presented with a post-op swelling, thus to counteract the swelling, we administer dexamethasone injection 8mg starting dose followed by $4 \mathrm{mg}$ twice daily for two days, with significant improvement noted in the patient. Dexamethasone showed significant effects in decreasing the levels of prostaglandin E2 (PGE2) and thromboxane B2 (TxB2), which are some of markers of the extent of inflammation [30].

Chemical conjunctivitis presented in $4.9 \%$ of the patients who underwent surgery. Conjunctivitis is an inflammatory disease characterized by conjunctival erythema, swelling, and discharge. In this study occurrence of chemical conjunctivitis was attributed to be caused by Povidone-Iodine solution which is used during skin preparation prior to surgery. Povidone-iodine has been associated with non-infective (sterile) conjunctivitis, probably because of its toxicity to the ocular surface [31]. Usually the condition is self-limiting and requires no treatment [32]; however since most patient complained of discomfort, Normal saline $0.9 \% \mathrm{w} / \mathrm{v}$ eye drops were used 3 - 4 times per day, which helped the patient to relieve discomfort.

Speech impairment presented in $14.7 \%$, and Functional impairment was evident in $23.5 \%$ of the patients, and these were associated with surgical procedures which led to total/partial mandibulectomy, partial glossectomy and/or partial maxillectomy. Patients with maxillofacial pathologies often face multiple functional problems encompassing mastication, swallowing and speech amongst others subsequent to surgical treatment. These functional problems occur as a result of alterations of anatomy within the maxillofacial region. Since speech, swallowing and mastication rely on an appropriate coordination of a series of rapid and complex neuromuscular actions, any anatomical variation can have a compromising effect [32] [33]. After surgery for tumors of the mandible, patients may exhibit a number of morphological deficiencies: the loss of facial and jaw structures, as well as sensory and motor innervation that may result in dysfunction of the masticatory system and temporomandibular disorders [34]. Discontinuity of the mandible after surgical resection or trauma destroys the balance and symmetry of mandibular function, which leads to altered mandibular movements and deviation of the residual segment towards the defect side, resulting in loss of occlusion on the unresected side [35]. Although objective functional and speech outcome following maxillofacial surgery had sometimes shown impairment compared with the normal situation, it gave high subjective patient satisfaction with function and speech.

Surgical procedures may involve removal of part or whole tissue from the body, subsequently leaving a defect at site, such defect if is on facial region, may exhibit significant facial profile deformity and thus leading to aesthetic impairment. Anti-esthetic impact of surgical defect in immediate post-operative stages is minimal .In current study, $16.7 \%$ of patient presented with aesthetic impairment following surgery. The aesthetic considerations are those pertaining to facial symmetry, loss of lower facial height, chin projection, and scars in the facial skin. Several studies report of aesthetic impairment following surgery in maxillofacial region though few report of prevalence [36]-[38]. In the current study, site of incision had a significant impact on the esthetic aspect, extra oral incisions caused more of esthetic impairment than intra oral, and this can be explained by the fact that, unlike intra oral incisions, extra oral are easily noticeable since they are easily visible hence raises concern.

Neurological deficit was noted in $7.8 \%(n=8)$ of the patients in this study. These deficits presented as either loss of sensation or loss of motor function to a given part, depending on the nerve affected. The neurologic deficit that was most common, included, hemi-facial paralysis after facial nerve sacrifice following parotidectomy, and numbness on the mentle region, following sacrifice of inferior alveolar nerve in either total or partial mandibulectomy.

Limitations of the present study were small sample size and single institutional study.

\section{Conclusion}

Most of post-operative complications are minor and manageable, however some complications are major and 
these can be subjective to underlying systemic condition. The occurrence of these complications not only increases duration of stay in the hospital, hence increasing cost of treatment, but also can affect the patient psychosocially. Therefore proper pre- and post-operative counseling is mandatory for both patients and their families regarding the anticipated complications, moreover the surgeons and anesthesiologists should also be prepared for the complications and be ready to provide necessary measures in combating them.

\section{References}

[1] https://en.wikipedia.org/wiki/Surgery

[2] Snell, R. (2008) Clinical Anatomy by Region. 8th Edition, 669.

[3] Bernardi, D., Barzan, L., Franchin, G., Cinelli, R., Balestreri, L., Tirelli, U., et al. (2005) Treatment of Head and Neck Cancer in Elderly Patients: State of the Art and Guidelines. Critical Reviews in Oncology/Hematology, 53, 71-80.

[4] Pai, P.S. (2010) Complications in Head and Neck Surgery. Otorhinolar Clinics: An International Journal, 2, 61-67.

[5] Shaheen, O.H. (1984) Problems in Head and Neck Surgery. Balliere Tindall, London.

[6] Evans, P.H.R. (1989) Complications in Head and Neck Surgery and How to Avoid Trouble. The Journal of Laryngology and Otology, 103, 926-929. http://dx.doi.org/10.1017/S0022215100110527

[7] Motamed, M.H. (2003) An Assessment of Maxillofacial Fractures: A 5-Year Study of 337 Patients. American Association of Oral and Maxillofacial Surgeons, 61, 61-64. http://dx.doi.org/10.1053/joms.2003.50049

[8] Van den Bergh, B., Karagozoglu, K.H., Heymans, M.W. and Forouzanfar, T. (2012) Aetiology and Incidence of Maxillofacial Trauma in Amsterdam: A Retrospective Analysis of 579 Patients. Journal of Cranio-Maxillo-Facial Surgery, 40, 165-169. http://dx.doi.org/10.1016/j.jcms.2011.08.006

[9] Ku, C.M. and Ong, B.C. (2003) Postoperative Nausea and Vomiting: A Review of Current Literature. Singapore Medical Journal, 44, 366-374.

[10] Kenny, G.N.C. (1994) Risk Factors for Postoperative Nausea and Vomiting. Anaesthesia, 49, 6-10. http://dx.doi.org/10.1111/j.1365-2044.1994.tb03576.x

[11] Voss, L.J., Sleigh, J.W., Barnard, J.P.M. and Kirsch, H.E. (2008) The Howling Cortex: Seizures and General Anesthetic Drugs. Anesthesia and Analgesia, 107, 1689-1703.

[12] Ferrer-Allado, T., Brechner, V.L., Dymond, A., Cozen, H. and Crandall, P. (1973) Ketamine-Induced Electroconvulsive Phenomena in Human Limbic and Thalamic Regions. Anesthesiology, 38, 333-344. http://dx.doi.org/10.1097/00000542-197304000-00006

[13] Constant, I., Seeman, R. and Murat, I. (2005) Sevoflurane and Epileptiform EEG Changes. Pediatric Anesthesia, 15, 266-274. http://dx.doi.org/10.1111/j.1460-9592.2004.01538.x

[14] Poulton, T.J. and Ellingson, R.J. (1984) Seizures Associated with the Induction of Anesthesia with Isoflurane. Anesthesiology, 61, 471-476. http://dx.doi.org/10.1097/00000542-198410000-00022

[15] Walder, B., Tramer, M.R. and Seeck, M. (2002) Seizure-Like Phenomenon and Propofol. A Systematic Review. Neurology, 58, 1327-1332. http://dx.doi.org/10.1212/WNL.58.9.1327

[16] International Association for the Study of Pain (1979) Subcommittee on Taxonomy. Pain Terms: A List with Definitions and Notes on Usage. Pain, 6, 249-252.

[17] Kehlet, H. (1997) Multimodal Approach to Control Postoperative Pathophysiology and Rehabilitation. British Journal of Anaesthesia, 78, 606-617. http://dx.doi.org/10.1093/bja/78.5.606

[18] Price, D.D., Hayes, R.L., Ruda, M. and Dubner, R. (1980) Spatial and Temporal Transformations of Input to Spinothalamic Tract Neurons and Their Relation to Somatic Sensations. Journal of Neurophysiology, 41, 933-947.

[19] Hansen, B.J. and Rosenberg, J. (1993) Persistent Postoperative Hiccups: A Review. Acta Anaesthesiologica Scandinavica, 37, 643-646. http://dx.doi.org/10.1111/j.1399-6576.1993.tb03781.x

[20] Mammen, E.F., Koets, M.H., Washington, B.C., Wolk, L.W., Brown, J.M., Burdick, M., et al. (1985) Hemostasis Changes during Cardiopulmonary Bypass Surgery. Seminars in Thrombosis and Hemostasis, 11, 281-292. http://dx.doi.org/10.1055/s-2007-1004382

[21] Matory, Y.L. and Spiro, R.H. (1993) Wound Bleeding after Head and Neck Surgery. Journal of Surgical Oncology, 53, 17-19. http://dx.doi.org/10.1002/jso.2930530107

[22] Nambu, J., Sugino, K., Oishi, K., Yano, M., Nishihara, M. and Dohi, K. (2013) Characteristics of Postoperative Bleeding after Neck Surgery. Surgical Science, 4, 192-195. http://dx.doi.org/10.4236/ss.2013.43036

[23] Poole, G.V. and Winston-Salem, N.C. (1985) Mechanical Factors in Abdominal Wound Closure: The Prevention of Fascial Dehiscence. Surgery, 97, 631-639. 
[24] Makela, J.T., Kiviniemi, H., Juvonen, T. and Laitinen, S. (1995) Factors Influencing Wound Dehiscence after Midline Laparotomy. The American Journal of Surgery, 170, 387-390. http://dx.doi.org/10.1016/S0002-9610(99)80309-2

[25] Meena, K., Ali, S., Chawla, A.S., Aggarwal, L., Suhani, S., Kumar, S. and Khan, R.N. (2013) A Prospective Study of Factors Influencing Wound Dehiscence after Midline Laparotomy. Surgical Science, 4, 354-358. http://dx.doi.org/10.4236/ss.2013.48070

[26] Paydarfar, J.A. and Birkmeyer, N.J. (2006) Complications in Head and Neck Surgery—A Meta-Analysis of Postlaryngectomy Pharyngocutaneous Fistula. Archives of Otolaryngology_Head and Neck Surgery, 132, 67-72. http://dx.doi.org/10.1001/archotol.132.1.67

[27] Dubay, D.A. and Franz, M.G. (2003) Acute Wound Healing: The Biology of Acute Wound Failure. Surgical Clinics of North America, 83, 463-481. http://dx.doi.org/10.1016/S0039-6109(02)00196-2

[28] Mangram, A.J., Horan, T.C., Pearson, M.L., Silver, L.C. and Jarvis, W.R. (1999) Hospital Infection Control Practice Advisory Committee. Guideline for Prevention of Surgical Site Infection. Infection Control \& Hospital Epidemiology, 20, 247-278.

[29] Owens, C.D. and Stoessel, K. (2008) Surgical Site Infections: Epidemiology, Microbiology and Prevention. Journal of Hospital Infection, 70, 3-10. http://dx.doi.org/10.1016/S0195-6701(08)60017-1

[30] Filho, J.R.L., Maurette, P.E., Allais, M., Cotinho, M. and Fernandes, C. (2008) Clinical Comparative Study of the Effectiveness of Two Dosages of Dexamethasone to Control Postoperative Swelling, Trismus and Pain after the Surgical Extraction of Mandibular Impacted Third Molars. Medicina Oral Patologia Oral y Cirugia Bucal, 13, E129-E132.

[31] Scott, W.J. and Eck, C.D. (2012) Povidone-Iodine and Ophthalmia Neonatorum. Ophthalmology, 119, 653-654. http://dx.doi.org/10.1016/j.ophtha.2011.11.037

[32] Chandler, J.W. and Rapoza, P.A. (1990) Ophthalmia Neonatorum. International Ophthalmology Clinics, 30, 36-38. http://dx.doi.org/10.1097/00004397-199030010-00018

[33] John, S., Hassuji, R.M. and Rajashekhar, B. (2011) Speech and Swallowing Outcomes in Buccal Mucosa Carcinoma. Indian Journal of Palliative Care, 17, 238-240. http://dx.doi.org/10.4103/0973-1075.92344

[34] Nastalska, E.M., Rolski, D., Kubik, K.R. and Janicka, J.K. (2006) Treatment Considerations for Mandibulectomy Patients with Temporomandibular Dysfunction. Acta Stomatologica Croatica, 40, 295-301.

[35] Beumer, J., Curtis, T.A. and Firtell, D.N. (1979) Maxillofacial Rehabilitation: Prosthodontics and Surgical Considerations. The CV Mosby Co., St Louis, 130-156.

[36] Paranque, A.R., Steve, M., Vazquez, L., Bolleyn, A., Roze-Pellat, M.A. and Ehrenfest, D.M. (2011) Esthetic and Functional Reconstruction of the Posttumoral Interrupted Mandible With Double-Barrel Fibular Free Flap Graft: Rationale for a Microsurgical and Prosthodontic Approach. Journal of Oral Implantology, 37, 571-577. http://dx.doi.org/10.1563/AAID-JOI-D-10-00060

[37] Moore, D.J. and Mitchell, D.L. (1976) Rehabilitating Dentulous Hemimandibulectomy Patients. The Journal of Prosthetic Dentistry, 35, 202-206. http://dx.doi.org/10.1016/0022-3913(76)90280-8

[38] Haraguchi, M., Mukohyama, H., Reisberg, D. and Taniguchi, H. (2003) Electromyographic Activity of Masticatory Muscles and Mandibular Movement during Function in Marginal Mandibulectomy Patient. Journal of Medical and Dental Sciences, 50, 257-264. 